\title{
MCORD - MPD Cosmic Ray Detector a new features
}

M. Bielewicz ${ }^{1,2, *}, M$. Milewicz-Zalewska ${ }^{2}$, M. Grodzicka-Kobylka ${ }^{1}$, S. Mianowski ${ }^{1}$, P. Sibczynski ${ }^{1}, L$. Swiderski ${ }^{1}$, T. Szczesniak $^{1}, M$. Linczuk $^{3}, D$. Wielanek ${ }^{3}, A$. Kisiel $^{3}$, G. Kasprowicz ${ }^{3}, K$. Pozniak ${ }^{3}, W . Z_{\text {Zabołotny }}^{3}, R$. Romaniuk ${ }^{3}, A$. Chłopik $^{1}, A$. Bancer ${ }^{1}$, $K$. Grodzicki ${ }^{1}, M$. Pietrzak ${ }^{1}, A$. Dudziński ${ }^{1}, E$. Jaworska ${ }^{1}, E$. Strugalska-Gola ${ }^{1}, M$. J. Peryt ${ }^{3,2}$, $M$. Rybczynski ${ }^{4}, Z$. Wlodarczyk ${ }^{4}, G$. Stefanek ${ }^{4}$, and $P$. Kankiewicz ${ }^{4}$

${ }^{1}$ National Centre for Nuclear Research, ul. Andrzeja Sołtana 7, 05-400 Otwock-Świerk, Poland

${ }^{2}$ Joint Institute for Nuclear Research, 141980 Dubna, Russia

${ }^{3}$ Warsaw University of Technology, Pl. Politechniki 1, 00-661 Warsaw, Poland

${ }^{4}$ Jan Kochanowski University, ul. Żeromskiego 5, 25-369 Kielce, Poland

\begin{abstract}
The main detector system at the Nuclotron-based Ion Collider fAcility (NICA) located in Dubna, Russia is the Multi-Purpose Detector (MPD). For better calibration reason, the MPD needs an additional trigger system for an off-beam calibration of MPD sub-detectors and for rejection (veto) of cosmic muons. The system should also be useful for practical astrophysics observations of cosmic showers. The consortium NICA-PL group defines goals and basic assumptions for the MPD Cosmic Ray Detector (MCORD). This article describes the conceptual design and simulation plans of the MCORD detector based on plastic scintillators with SiPM photodetectors and electronic digital system based on the MicroTCA crate.

Keywords: MCORD, NICA, MPD, FPGA, Cosmic Ray detector, MicroTCA, trigger system, Cosmic Shower.
\end{abstract}

\section{Introduction}

A new big accelerator complex is currently under construction at the Joint Institute for Nuclear Research in Dubna created in order to study properties of dense baryonic matter (Fig. 1). One of the main parts of the Nuclotron-based Ion Collider fAcility (NICA) [1] is the MultiPurpose Detector (MPD) [2]. The MPD detector was designed to track products emitted during ion-ion collisions that will take place in one of two crossing points of beams accelerated in opposite directions. The MPD is placed in a dedicated experimental hall and its operation is maintained by a Slow Control System [3-5] installed in racks located on a platform placed by the MPD (Fig. 2).

The role of the MPD is to provide the information necessary for the reconstruction of each event. However, cosmic muons coming from cosmic showers are one of the main sources of background in the high-energy physics instrumentation as they generate similar or even identical signals as charged particles produced during collisions or during decays of neutral products. Therefore, the MPD needs an additional triggering system that could be used to discriminate signals induced by cosmic showers (mainly muons). Cosmic showers are created by primary cosmic particles (mainly protons) interacting with atmospheric particles and

\footnotetext{
*e-mail: marcin.bielewicz@ncbj.gov.pl
} 


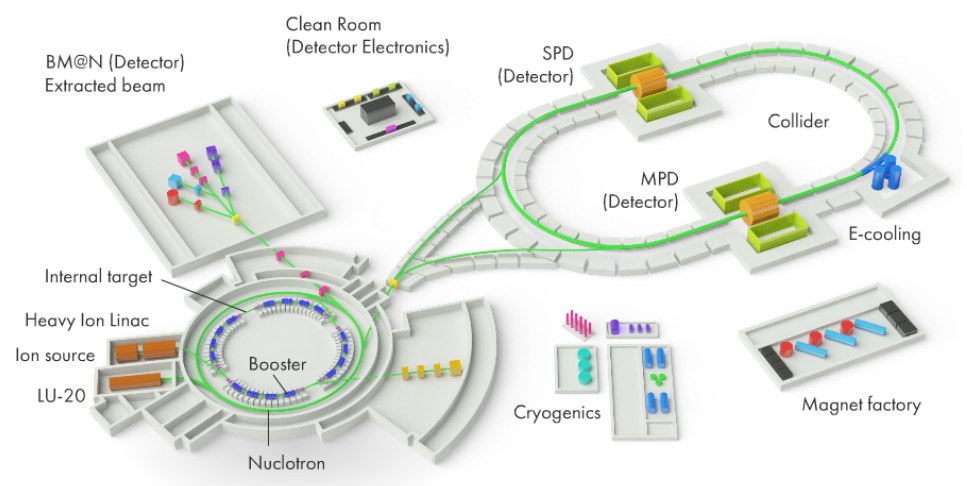

Figure 1. The NICA accelerator complex [1]

creating cascades (Fig. 3). This system could also be used for an off-beam calibration of the MPD (mainly the TPC and TOF detectors) response to muons and to provide data for astrophysics measurements of high-energy muon showers. In order to enrich the MPD with these features, we propose to surround the MPD cylinder with a cosmic-ray detector called MCORD (Fig. 4). A similar system (ACORDE) was constructed at CERN for the ALICE detector, Fig. 5 [6]. The main difference is that ALICE with the LHC ring is located deep underground $(55 \mathrm{~m})$ whereas the MPD is located on the ground level. The specific location of ALICE and ACORDE forms a natural barrier that filters low-energy muons (threshold 16 $\mathrm{GeV}$ ) and most of other charged particles and gamma rays. Moreover, detected muons come only from the zenith direction. The MPD with MCORD located on the ground level will detect muons coming from all directions between zenith and horizon filtered to some extent only by concrete walls of the experimental hall.

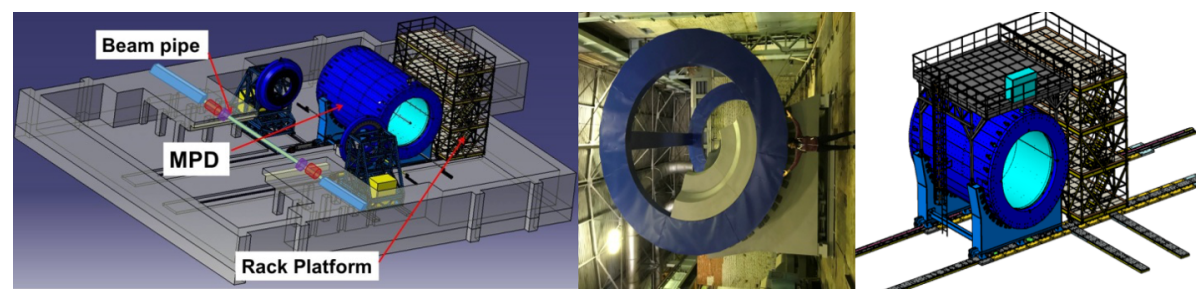

Figure 2. On the left: Room for the MPD detector. In the center: The real size model of the MPD. On the right: the MPD detector and the Platform for the MPD electronic racks [2], [1]

MCORD should be able to provide the information on the time, position, direction and amplitude of the signal induced by particles passing through the scintillators. Based on this data, the calibration of the TPC and TOF detectors should be possible. The MCORD signals should also serve as a veto trigger for a cosmic shower to avoid the misinterpretation of events from ion-ion collisions. Since MCORD will cover a substantial fraction of the MPD volume, it could also be very useful for the observation of cosmic showers initiated by extremely high energy primary particles coming especially from the horizon direction. The MCORD detector will be designed and constructed by the Polish consortium NICA-PL joining several scientific institutions. Within the course of the project, there will be several work packages 


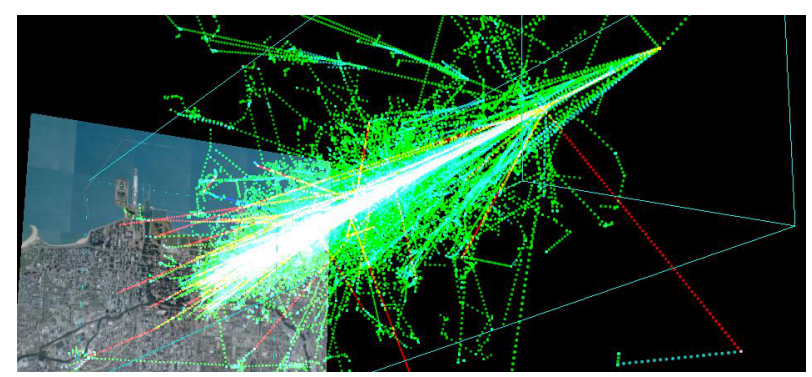

Figure 3. Cosmic ray air shower created by a $1 \mathrm{TeV}$ proton hitting the atmosphere $20 \mathrm{~km}$ above the Earth. The shower was simulated using the AIRES package [7]

devoted to the MCORD detector design and construction. Besides the experimental work, the choice of optimal detectors will be supported by simulations performed using the MCNPX, GEANT4, CORSIKA, and Showersim.

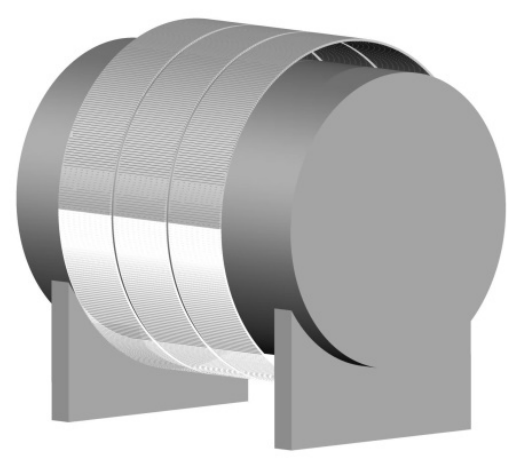

Figure 4. Conceptual design of the MCORD detector surrounding the MPD detector at the NICA complex

Following to preliminary discussions between partners involved at the project stage, simple plastic scintillators with light readout were chosen as the most suitable candidates for a detector module. At the first stage of the project, experiments and simulations will be done to choose the optimum shape of scintillators (length, width and thickness), the configuration of photodetectors and readout electronics. Basing on the output of this study, the MCORD shape and size will be proposed taking into account requirements from the MPD users, the performance of the MCORD system and especially the production cost of the device. At the second stage of the project (Demonstrator construction), a few (2-4) detector modules (Fig. 6) will be provided for calibration and testing of the TOF and TPC detectors. Each MCORD module will consist of 18 plastic scintillators with 36 measurement channels. The final stage of the project involves the construction of the full-size MCORD detector around the MPD surface. 

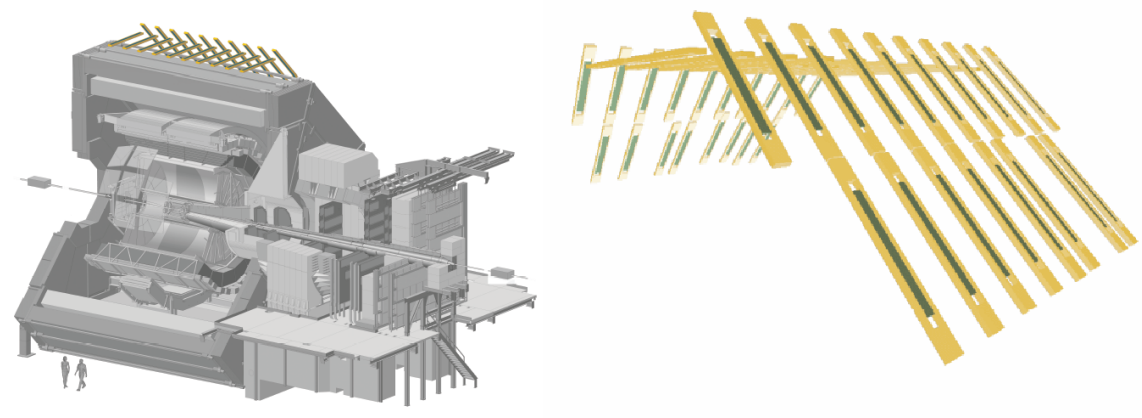

Figure 5. On the left: ALICE with the ACORDE detector on the top (yellow). On the right: the ACORDE cosmic ray detector [6]

\section{MCORD system description}

The MPD is an example of a large size scientific device ( $8 \mathrm{~m}$ in length and $6 \mathrm{~m}$ in diameter). Therefore, the MCORD detector needs to be designed using relatively cheap and efficient materials. The most common material for muon detection is a plastic scintillator. Since the MPD detector comprises a large magnet, around which the MCORD detector should be installed, we propose to use photodetectors insensitive to a magnetic field. Such feature is offered by semiconductor detectors like silicon photomultipliers (SiPMs), also known as multi-pixel photon counters (MPPCs). The main features of the MCORD detector should be $2 \mathrm{D}$ positional sensitivity, precise timing information and cost-effectiveness (low price per unit module). However, fulfilling these requirements pose contradictory constraints on the detector design. Therefore, not only the optimization but also some trade-off on the detector performance is necessary. Concerning the detector cost, simple plastic scintillators are one of the best candidates for MCORD detection medium as they are one of the cheapest charged particles sensitive materials $[8,9]$. To ensure enough positional sensitivity we propose to use long, rather thin narrow slabs with double-sided photodetectors (SiPMs). At present, the most probable scintillators size will be $1500 \times 10 \times 10 \mathrm{~mm}$ or $1500 \times 10 \times 25 \mathrm{~mm}$. The time difference in the light propagation through the plastic will be used to reconstruct the particle interaction point along the scintillators module, whereas the width and thickness of the slab will give limits on the muon track point in the plane perpendicular to the long detector axis. The second possibility is to use scintillators with two or three fibers to make better position resolution in the second axis (width). The use of fiber makes it possible to use much smaller SiPMs $(1 \times 1 \mathrm{~mm})$, which are much cheaper than the bigger ones (e.g. $25 \times 25 \mathrm{~mm})$. On the other hand, scintillators production cost will be higher in this case. It is not yet decided which option will be better for the MCORD detector.

During the development of the complicated and large systems like MCORD, simulations of the muon interactions with scintillators, building walls, and other detector elements play an important role in the estimation of the system performance and cost reduction. For that purpose, several parameters need to be calculated using simulations:

- energy distribution of cosmic muons as a function of emission angle,

- composition of cosmic particles as a function of emission angle,

- simulation of the muons deposition energy through the concrate walls, other MPD subdetectors and the MPD yoke and cover. 
For the simulation of the energy deposition and optimization of the systems geometry tracking and TOF characteristics, the MCNPX (Monte Carlo N-Particle Transport Code System for Multiparticle and High Energy Applications) [10] and GEANT4 (toolkit for the simulation of the passage of particles through matter) [11] codes will be used. The energy and angle distribution and composition of the cosmic radiation will be calculated using specialized simulation codes such as CORSICA and Showersim.

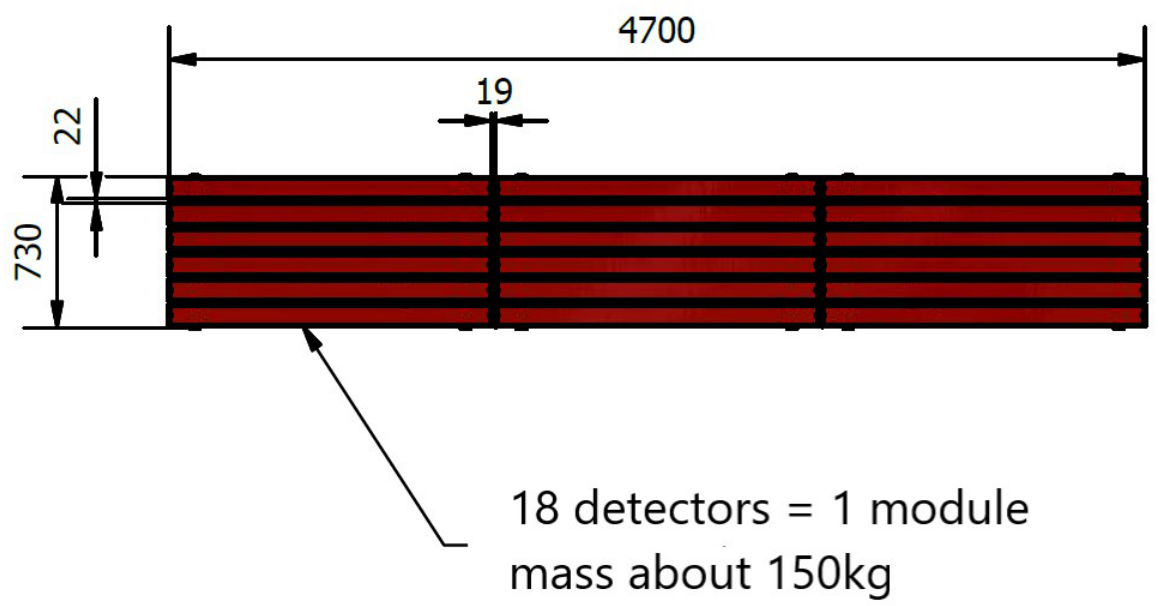

Figure 6. The MCORD detector modules consist of three sections, six scintillators each. The modules and sections can be separated from each other.

The MCORD detector modules (Fig. 6) will be arranged in a barrel shape around the cover of the MPD detector (Fig. 7). Depending on the granularity of the MCORD scintillators, it will comprise from few hundred up to few thousand of double-side readout detectors. The scheme of the signal (analog) readout path is presented in Fig. 8.
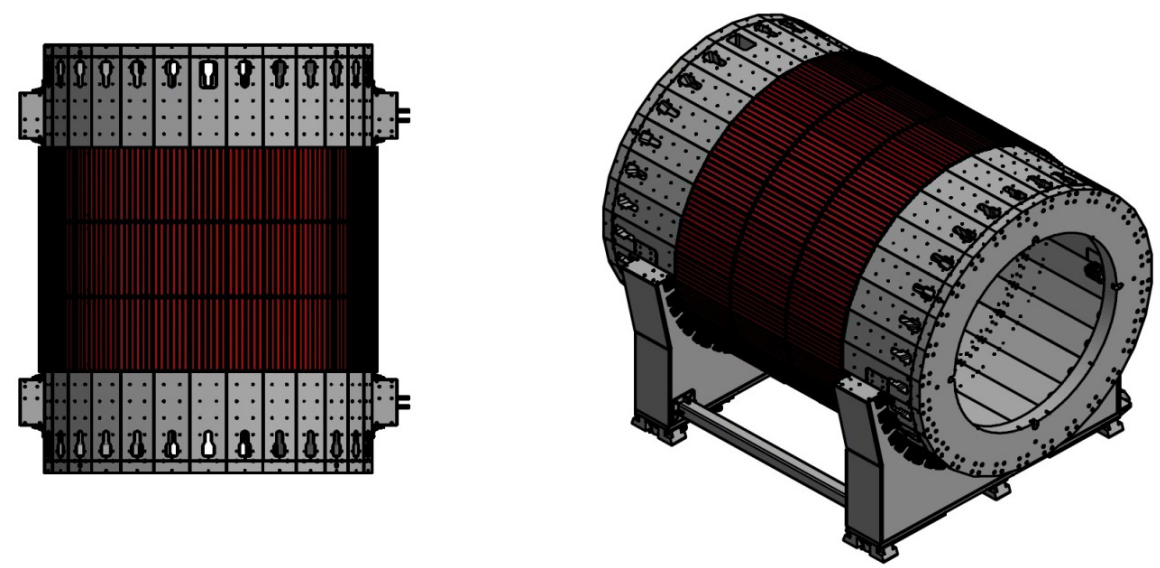

Figure 7. Basic version of the MCORD design surrounding the MPD detector at the NICA complex 


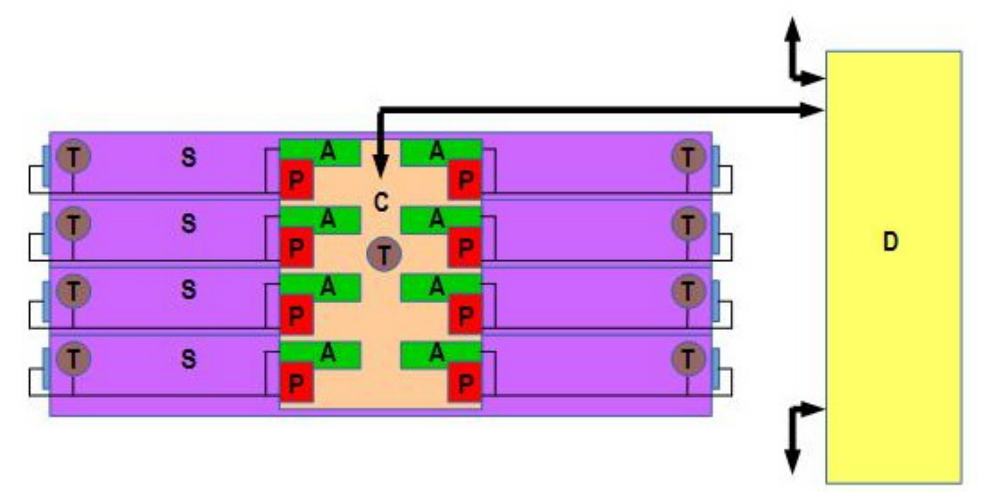

Figure 8. Conceptual design of the MCORD detector readout section. Legend: S (violet) - plastic scintillator, (blue-both ends of scintillators) - SiPM, P (red) - power supply with the temperature compensation circuit, T (brown) - temperature sensor, A (green) - amplifier, D (yellow) - MicroTCA digital system, C (orange) - Analog Front End Module

When a muon hits a scintillator it creates a quant of light. A SiPM activated by photons generates electrons and an electrical output proportional to the number of detected photons. SiPMs are characterized by a high gain $\left(10^{5} \div 10^{6}\right)$, single photon detection capability, fast counting and low-bias voltage $(\sim 30 \mathrm{~V} \div 70 \mathrm{~V})[12]$. Insensitivity to the magnetic field is one of the greatest advantages of SiPMs over classical PMTs. However, there are also disadvantages, like SiPM gain sensitivity to temperature changes, which requires the application of temperature compensation circuits coupled with power supplies. The analog output signal from a SiPM is amplified and shaped by an amplifier in Analog Front End Module and subsequently is sent to the MicroTCA System (MicroTCA $\AA$ is a modular, open standard for building high performance switched fabric computer systems in a small form factor) [15]. One MicroTCA System receives signals from many Analog Front End Modules. A relevant controller will be designed to maintain the operation of the detector power supplies, temperature compensation circuits, amplifiers, and digitizers.

Each SiPM detector is directly connected to the Analog Front-End Module (AFEM). This module consists of a very simple amplifier with pre-amplifier and shaper. The shaper is dedicated to pulse formation. Pulses after this module have a reduced bandwidth and thus increased duration. The bandwidth limitation reduces hardware requirements for readout equipment and minimizes RF interferences. AFEM will be located as close to the detector as possible. For this reason the AFEM hardware must be radiation resistant. Moreover, AFEM consists of power supply for SiMP detectors with sensor temperature compensation circuit and pre-amplifier. This part must be located as close to each SiPMs as possible. Analog signals are transmitted to the ADC located on the FMC boards located in the MTCA processing system. The signals are transmitted via standard VHDCI connectors and a 24 or 32-channels cable. The signal processing system is based on the Micro TCA.4 standard (Fig. 9). This is a modular system for acquisition, control, management and data processing. Version 4 of this standard was developed and standardized by DESY. The main advantage of this standard is the high-speed backplane up to $40 \mathrm{Gbit} / \mathrm{s}$ based on gigabit links. The chassis integrates the embedded computer, management controller and PCIe Gen 3 and Ethernet hub. 


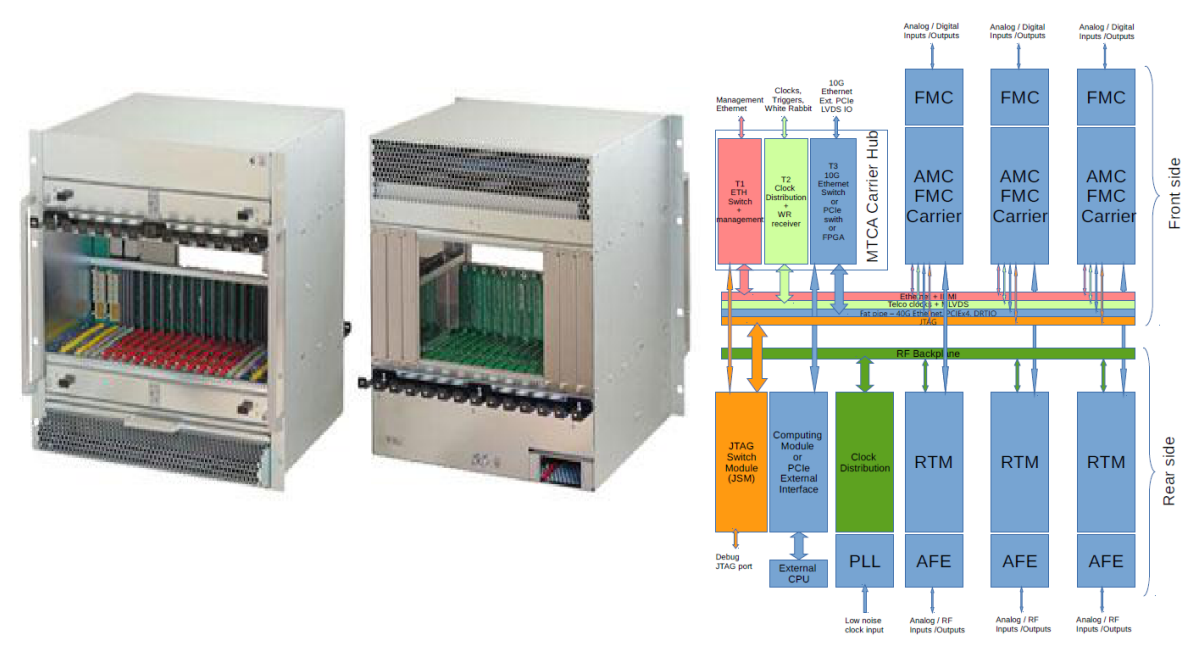

Figure 9. On the left: The Micro TCA.4 crate. On the right: An architecture of MicroTCA.4 standard ver4

The TCA standard allows one to install up to 12 cards in the AMC (Advanced Mezzanine Card) standard. For MCORD, the industry standard AMC-FMC carriers will be used. The FMC (FPGA Mezzanine Card) is an ANSI standard that provides a standard mezzanine card form factor, connectors, and modular interface to the FPGA located on a baseboard. The Micro TCA architecture shown in Fig. 7 has many advantages. The basic elements of the system are highlighted in blue. In addition to the FMC and AMC-FMC modules, there is the MCH (MTCA Carrier Hub) and the optional Computing Module. The MCH has gigabit ports that enable connection of multiple cassettes into one system. The RTM and AFE modules can be optionally used for precise analog signal processing and will not be used in this experiment. A computing module is not foreseen for MCORD either. The other modules are optional and can be used for future upgrades of the system. The T2 Clock Distribution module enables the use of the White Rabbit standard [16] to synchronize multiple AMCs and MTCAs with sub-ns accuracy. The JTAG Switch Module allows remote debugging of FPGA devices. This is useful during development of the software, it enables remote access to the system installed in the tunnel or experimental area under radiation conditions.

A complete system architecture projected and dedicated for the MCORD system is shown in Fig. 10. The whole system will consist of four MicroTCA crates (14U size each). Signals from the detector are processed locally by the AFE modules and transmitted to the FMC cards, which provide AD conversion. There are two FMC cards on each AMC carrier board. Up to 12 AMC cards on each MicroTCA crate can be installed. The data after processing in the AMC FPGA is transferred using the MTCA backplane to the MCH module. The MCH contains a large Kintex FPGA, which performs further processing. There are two trigger levels. First trigger level is implemented in the AMC FPGA. The Second level trigger is implemented in the FPGA on the MCH module.

One of the three MCH FPGAs serves also as a hub that receives data from another MTCA crates using the 10GBit serial link. The number of input channels of the whole system depends on the bandwidth of ADCs. The idea is to reduce the ADC sampling speed to reduce the amount of data that needs processing. Several parameters of detector pulses are calculated using the algorithm implemented in the FPGA (e.g. time of flight, the time difference 


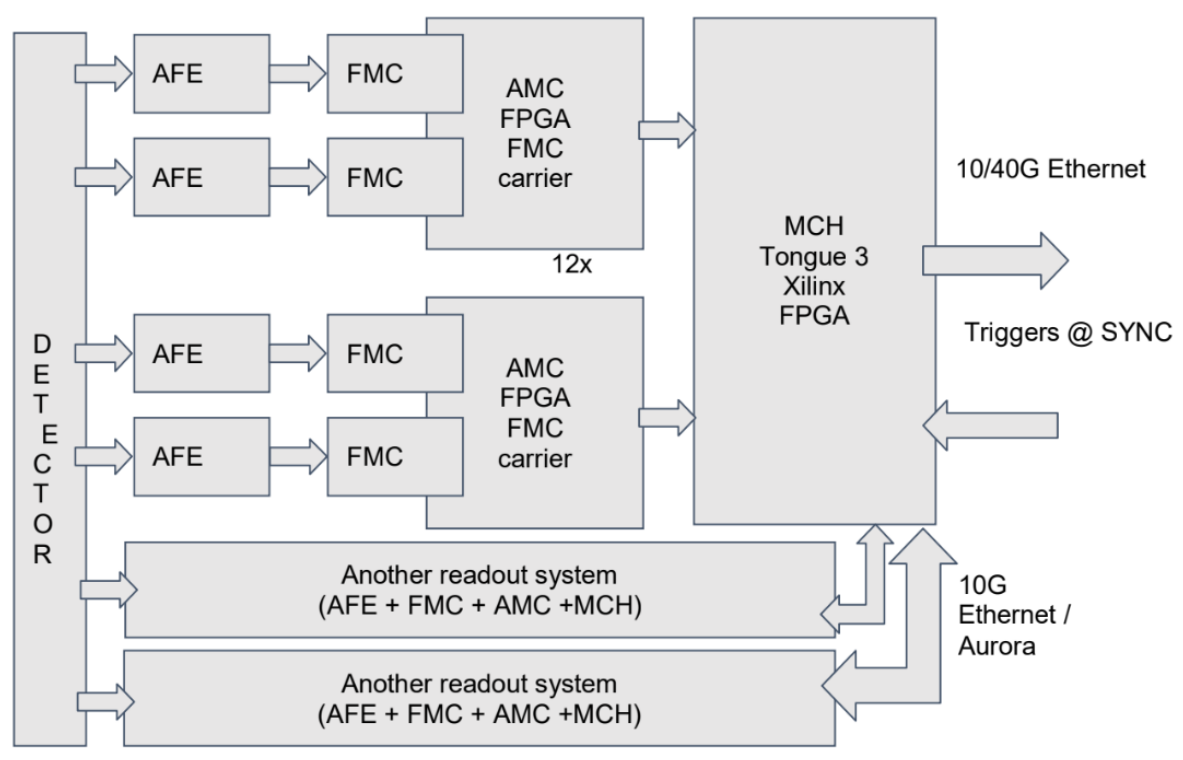

Figure 10. System architecture based on the MicroTCA standard

between channel pairs, energy). Since the shape of the pulse is known in advance, on-line deconvolution algorithms can be used to calculate the parameters with accuracy much higher than sampling period.

There are few options that depend on selection of the FMC-ADC cards:

- 8 channels/14bit @ 250MS/s (highest performance),

- 16 channels/14bit @ 125MS/s (shown in Fig),

- 24 channels/14bit @ 80MS/s,

- 32 channels/14bit @ 50MS/s (lowest cost).

The lowest cost option is $50 \mathrm{MS} / \mathrm{s}$ which provides 32 channels. This option can reduce the number of the MicroTCA crates required for MCORD. The highest performance option is $250 \mathrm{MS} / \mathrm{s}$ and it offers the best quality of the input signals processing. Our present choice is $125 \mathrm{MS} / \mathrm{s}$ option. Depends on required signal processing capability, the number of channels per one MicroTCA system is:

- at 250MS/s: 192 channels/crate,

- at $125 \mathrm{MS} / \mathrm{s}: 384$ channels/crate,

- at 80MS/s: 576 channels/crate,

- at 50MS/s: 768 channels/crate.

\section{FPGA based muon trigger}

The muon trigger, implemented in the FPGA, will use signals produced by the photondetectors connected at both ends of the scintillator strips. The signals will be shaped and converted to the digital form using the ADC converters. Depending on the required analog signal bandwidth, 14-bit resolution ADCs with $125 \mathrm{MS} / \mathrm{s}$ will be used. 
The digitized signal will be processed in the FPGA to calculate the time and amplitude of signals at both ends. The position of the hit along the scintillator will be calculated from the time difference between those signals. Possibility to use the amplitude difference and constant fraction discriminator will be also investigated. If the extremely high precision position measurement is needed, it should be possible to provide the additional high-speed digital channel with the FPGA-based TDC.

The track and direction of the muon will be calculated in the FPGA basing on coincident hits from different detector layers. The fast and low latency process of a muons trajectory identification will be based on the muon trajectory processor original concept for the Overlap Muon Track Finder [15], [16] in the CMS muon trigger system [17].

\section{Summary}

This work is an example of the activities of the Consortium NICA-PL. The paper presents the simple concept of building a universal, reconfigurable, fast and modular cosmic muon trigger system. MCORD muon trigger is dedicated to the NICA-MPD detector. The detection part is based on low cost, plastic scintillators with SiPMs photodetectors for the light readout. The MTCA standard with the back plain version 4.0 designed specifically for the use in HEP experiments will be used for the construction of the device. The applied solution is based on the commonly used apparatus used at the CERN and GSI. It has been proposed to use exchangeable analog and digital modules including primarily FMC blocks containing appropriately matched A/D converter circuits. The proposed hardware solution allows for an easy modification of the system in terms of the required number of channels. The key issue is to use the FPGAs for the fast, low-latency, numerically advanced signal processing from detectors in order to accurately determine the time and place of passage of the ionizing particle by a scintillator. The use of layers of the FPGAs placed on the AMC and MCH modules, respectively, enabled the functional structuring of the muon trigger by separating the main stages of the process.

A cosmic ray detector is necessary for good calibration of the TPC, TOF and ECAL, MPD detectors before completion of the MPD, and it will be very helpful for better calibration of the TPC and TOF detectors before each future experimental session. In addition, MCORD can be used for astrophysics observations similar to past collider experiments. In our case, especially for studies of near horizontal muon bundles (research of the main trivial mechanism of the multi-muon event generation (EAS muons).

The proposed approach will allow one to obtain high-precision and quality measurement data, despite the assumed limit of analogue transmission bandwidth in order to minimize the level of interference, as well as the construction costs of a complete device. The projected cosmic ray detector will be designed to have the required time resolution and position accuracy. Our team has a realistic plan and is capable of building this cosmic ray detector.

\section{References}

[1] http://nica.jinr.ru/complex.php

[2] V. Golovatyuk, V. Kekelidze, V. Kolesnikov, O. Rogachevsky and A. Sorin, Eur. Phys. J. A 52, 212 (2016)

[3] D. Dabrowski et al., Act.Phys.Pol.B 92(2), 203 (2016)

[4] M. J. Peryt, T. Traczyk, Act.Phys.Pol.B 92(2), 293 (2016)

[5] K. Roslon et al., Act.Phys.Pol.B 92(2), 299 (2016)

[6] A. Fernandez, S. Kartal, C. Pagliarone, arXiv:physics/0612051 [physics.ins-det] 
[7] https://en.wikipedia.org/wiki/Air_shower_(physics)

[8] https://eljentechnology.com/products/plastic-scintillators/ej-200-ej-204-ej-208-ej-212

[9] https://www.crystals.saint-gobain.com/products/bc-408-bc-412-bc-416

[10] C.J. Werner, J.S. Bull, C.J. Solomon et al., MCNP6.2 Release Notes, LA-UR-18-20808 (2018)

[11] S. Agostinelli et al., Nuclear Instruments and Methods in Physics Research A 506, 250303 (2003)

[12] M. Grodzicka-Kobylka, T. Szczesniak, M. Moszyński, Nucl. Instrum. and Meth. in Phys. Res. A 856, 53 (2017)

[13] https://www.picmg.org/openstandards/microtca/

[14] https://www.ohwr.org/projects/wr-std/wiki

[15] W.M. Zabołotny et al., Journal of Instrumentation 12, C01050 (2017)

[16] W.M. Zabolotny, A. Byszuk, Journal of Instrumentation 11, C03004 (2016)

[17] U. Bhawandeep et al., Journal of Instrumentation 12, 1-122 (2017) 